\title{
OFCS: Optimized Framework of Compressive Sensing for Medical Images in Bottleneck Network Condition
}

\author{
Lakshminarayana M. ${ }^{1}$, Mrinal Sarvagya ${ }^{2}$ \\ ${ }^{1}$ Department of ECE, Visvesvaraya Technological University, India \\ ${ }^{2}$ School of ECE, REVA University, India
}

\section{Article Info \\ Article history: \\ Received Mar 10, 2018 \\ Revised June 13, 2018 \\ Accepted June 28, 2018 \\ Keyword: \\ Compression \\ Compressive sensing \\ Medical image \\ Normalization \\ Region of interest \\ Transform}

\begin{abstract}
Compressive sensing is one of teh cost effective solution towards performing compression of heavier form of signals. We reviewed the existing research contribution towards compressive sensing to find that existing system doesnt offer any form of optimization for which reason the signal are superiorly compressed but at the cost of enough resources. Therefore, we introduce a framework that optimizes the performance of the compressive sensing by introducing 4 sequential algorithms for performing Random Sampling, Lossless Compression for region-of-interest, Compressive Sensing using transform-based scheme, and optimization. The contribution of proposed paper is a good balance between computational efficiency and quality of reconstructed medical image when transmitted over network with low channel capacity. The study outcome shows that proposed system offers maximum signal quality and lower algorithm processing time in contrast to existing compression techniuqes on medical images.
\end{abstract}

Copyright (C) 2018 Institute of Advanced Engineering and Science. All rights reserved.

\section{Corresponding Author:}

Lakshminarayana M.,

Department of ECE, Visvesvaraya Technological University,

Belgaum, Karnatak, India.

Email: lakshminarayana.m.2015@ieee.org

\section{INTRODUCTION}

The significant of radiological image has dependably been held high in medicinal services area as it is one of the most effortless and non-obtrusive component to analyze illnesses [1]. A typical and mid-level healthcare services unit may require performing stockpiling of high number of radiological images for future reference. In the meantime, telemedicine will likewise require transmitting such restorative images starting with one then onto the next end over regular system [2],[3]. Such system have regularly bring down asset accessibility and also insignificant channel limit, which offer ascent to corruption of received image alongside nature of administration issue. The prime explanation for this is the span of the medicinal image.

A typical Computed Tomography (CT) examine image extends between 2048x2048 pixels measure [4]. In the meantime, a normal size of multi-cut CT filters image ranges from 131 Megabytes to 2 Gigabytes. Accordingly, more the modern form of radiological image more is the measure of it. Such size amasses to exponentially higher degree, which are greatly testing to complete transmission on higher-limitation systems. One successful approach to take care of the issue of capacity and transmission is to perform compression. At present there are different types of pressure calculation; in any case, lossy and lossless is the prime classification of it. Both the plans have their own particular significant points and also inconveniences with regards to viability of the pressure procedure. A superior arrangement in these respects is to perform compressive sensing. Each procedure applies its calculation on the entire segment of the chosen input image. Such component is not adaptable and many-sided quality increments with the expansion of image size. Then again, compressive sensing doesn't utilize the entire image however just plays out a one of a kind inspecting process which guarantees better remaking process. Hence, conditions of computational assets are decreased 
and it diminishes the extent of information image without unfavorably influencing the flag quality at the recipient end. Tragically, there is additionally an entanglement related with compressive detecting e.g. i) it is a lossy pressure strategy and consequently the flag quality likewise depends of different questionable system parameters that can additionally debase the flag quality amid image transmission and ii) there is no standard component to guarantee uniform testing rate for various sorts and modalities of the medicinal images.

In this manner, there is a need of study that plays out a successful image sensing by upgrading the compressive detecting methods. While taking a shot at pressure, it is not attainable for addressing different system related issues those outcomes in flag declination, yet it is practical to address asset related issue. At present, the usage procedures towards pressure concentrates more on size decreasing on entire image but doesn't stress on the computational efficiency. In the region of medicinal diagnosis, determination must be kept up as high as conceivable particularly in the remade image. There is likewise another neglected issue related with existing pressure system i.e. doesn't considers the complexities that could emerge from the device. At present, various wireless communication devices have already being in practice in healthcare units where the physician can carry such device on the go. Such device doesn't have enough computational capability and is sustained by battery. Therefore, keeping future application in mind, compressive sensing is the better alternative of compression technique for medical images. The proposed manuscript discusses about a novel framework towards optimizing the compressive sensing performance. The prime emphasis is to enhance the computational efficiency associated with it. Section 1.1 discusses about the existing literatures where different techniques are discussed for compressive sensing in association with medical image compression schemes followed by discussion of research problems in Section 1.2 and proposed solution in 1.3. Section 2 discusses about algorithm implementation followed by discussion of result analysis in Section 3. Finally, the conclusive remarks are provided in Section 4.

\subsection{Background}

Our prior study has already discussed about various research contribution towards compressive sensing [5] therefore, we upgrade more about existing literatures in this section. Ambrosio et al. [6] have investigated the compressive sensing sensing with respect to security on image. Kavitha et al. [7] have implemented compressive sensing for the medical images considering random sampling. Usage of compressive sensing towards medical imaging is also seen in the work of Bevacqua et al. [8] using threedimensional geometry. Similar trend of work is also carried out by Foroozan and Sadeghi [9] using dictionary of wave atom. Chen et al. [10] have used compressive deconvolution for improving the medical images during compression using directional approach of multipliers. Guo abd Abbosh [11] have investigated compressive sensing towards identification of stroke using head imaging captured from microwave antenna. Baldassare [12] introduces a learning-based technique for increasing the signal power while performing compressive sensing on medical image. Jelena [13] have used compressive sensing to minimize the sampling of the data from medical images for enhanced version of reconstructed image. Yang et al. [14] have used Shearlet transform as well as compressive sensing toward medical image compression. Kustner et al. [15] have presented cartesia approach of subsampling for enhancing the performance of compressed sensing using partial acquisition of Fourier transform on medical image.

$\mathrm{Li}$ et al. [16] have investigated the usage of compressive sening on medical image reconstruction using measurement matrix for sparse reconstruction of an image. Panic et al. [17] have presented a reconstruction algorithm using markov random approach in order to design a clustering mechanism over medical images. Sa [18] have implemented an enrollment process using SIFT approach and compressive sensing using experimental approach. Sharma et al. [19] have introduced a technique where compressive sensing is used over ultrawideband signal. Xu et al. [20] have dicussed about an aggregation scheme of adaptive nature where the sparsity factor is determined dynamically depending upon the fluctuation of the signal. Wang et al. [21] have investigated toward signal acquisition process for developing a sampling matrix of optimal booloean type in order to enhance the recovery process of the biomedical signal. Zhang et al. [22] have applied compressive sensing as well as wavelet tree mechanism in order to perform reconstruction of a biomedical image. Study towards compressive sensing is also carried out towards medical image by Zhang et al. [23] where the technique also presents a thresholding scheme for implementing iterative wavelet schemes. Zhao et al. [24] have presented an algorithm for performing reconstruction of video signal using compressive sensing.

Usage of compressive sensing towards medical image was seen in the work carried out by Xi et al. [25] on real-time dataset. Therefore, it can be seen that there are various technical contribution towards enhancing the performance of the compressive sensing in most recent time. Mesra et al. [26] proposed a new image compression techniques using reversible mapping lossless techniques. This scheme optimizes the size of the image as well as the compression ratio to speed up the compression and reconstruction methods. This cyclic reversible scheme uses the Huffman encoding scheme to encode the compressed data at the transmitter 
side. Setyaningish et al. [27] presented a review research article based on the hybrid compression images using different conventional lossy and lossless compression schemes. It techniques improves the better visual quality of reconstructed images and gives good compression ratio. This hybrid techniques speed up the processing time of compression as well as better PSNR values. Suma et al. [28] designed a novel image compression for medical images using Urdhava Tiryakbhyam schemes. Here author introduced most advanced scheme is related to Vedic mathematics techniques. it enhances the quality of image as well as it reduces the power required to compute the compression of medical images. The performance of the image compression is evaluated using PSNR and compression ratio. All the above discussed techniques have their associated limitations apart from its advantages. The next section discusses about the research problems.

\subsection{Research Problem}

The significant research problems are as follows:

a. Majority of the existing approaches uses conventional compressive sensing without much inclusion of novelty towards optimization.

b. Existing studies doesn't focus on applying compressive sensing compatible for transmitting the signal over a network with low channel capacity.

c. The performance of compressive sensing could be further enhanced using random sampling process prior to implement it which is not found in existing system.

d. There are least number of benchmarked studies towards claiming the robustness of compressive sensing on medical image.

Therefore, the problem statement of the proposed study can be stated as "It is highly computationally challenging task to formulate an optimization towards compressive sensing performance with good balance between computational efficiency and quality of signal reconstruction."

\subsection{Proposed Solution}

The proposed study is an extension of our prior studies where the compressive sensing have been focused on individual cases [29]-[34]. The prime aim of the proposed study is to evolve up with a novel framework that perform optimization over the performance compressive sensing for assisting the multimedia files to be transmitted over a network with low channel capacity. The proposed system uses analytical research methods in order to develop and implement the algorithms. The schematic diagram is shown as Figure 1.

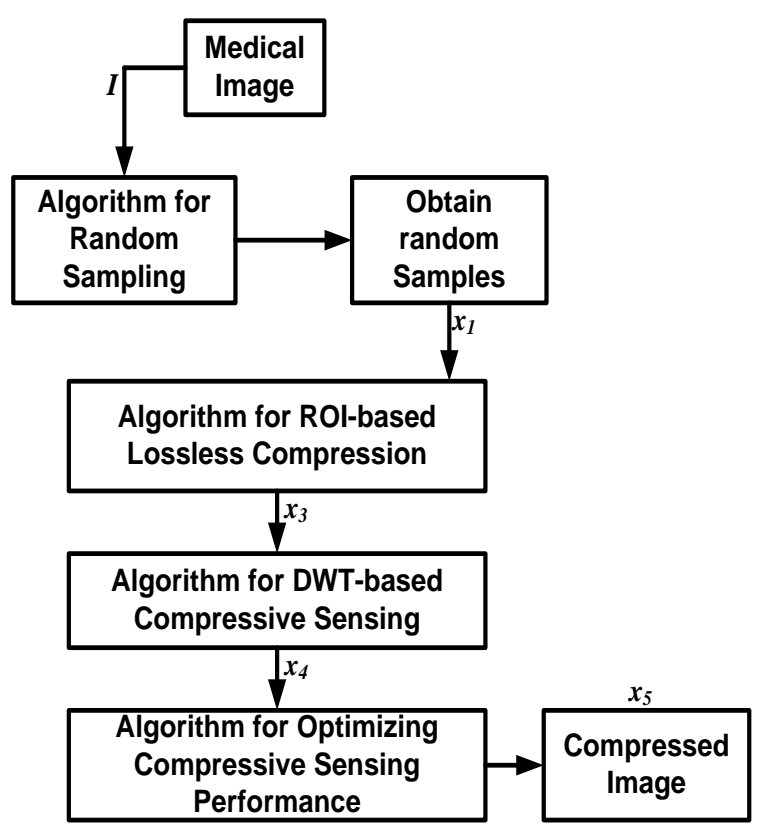

Figure 1. Schema of proposed methodology

The proposed algorithm is implemented with an aid of 4 unique algorithms that are essentially meant for enhancing the compression performance. The proposed system takes the input of medical image and extracts random samples which are further subjected to lossless compression on its Region of Interest 
(ROI). Discrete Wavelet Transform (DWT) is also used for extracting the signal coefficient that will assists the system to maintain minimal weight of pixel feature with faster processing. Finally, a hybridized algorithm is implemented by combining both lossless and lossy compression scheme in order to ensure that both the significant region as well as non-significant region is suitable compressed. The next section discusses about the algorithm implementation.

\section{ALGORITHM IMPLEMENTATION}

This section discusses about the algorithm design for accomplishing the research contribution discussed in prior section. It should be noted that proposed technique offers multiple series of operation in order to perform better optimization to compressive sensing using 4 sequential algorithms that are discussed as following:

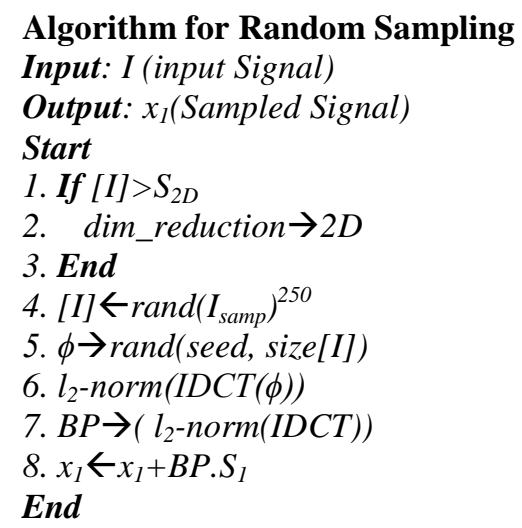

This algorithm is responsible for performong random sampling of the multimedia file $I$ so that the sampled outcome could be transmitted effectively over the peak traffic condition. The algorithm reads the input signal $I$ and compares its dimension with the two dimensional signal $\mathrm{S}_{2 \mathrm{D}}$ (Line-1). If the input signal is found of more dimension than it is subjected to dimensional reduction process back to two dimension (Line2). The algorithm than extracts 250 samples from the input signal in a random process (Line-4) that is followed by computation of measurement matrix $\phi$ that is dependent on seed and size of input signal (Line5). This is further followed by $l_{2}$ normalization using inverse discrete cosine transform (Line-6) followed by computation of basis pursuit function (Line-7) that finally results in a random sampled signal $\mathrm{x}_{1}$. The contribution of this algorithm is faster computation of the sampling for large multimedia files over peak traffic condition with low channel capacity. After the random samples are collected than it is further subjected to lossless compression using next algorithm as shown below:

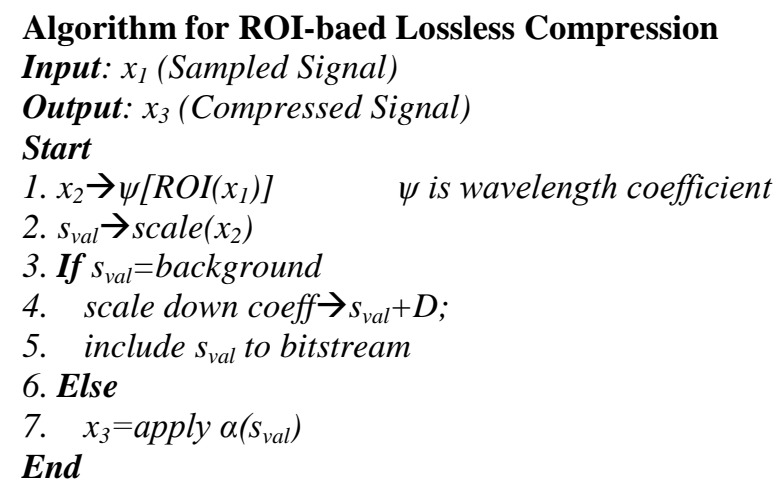

This algorithm is particularly constructed to resist the signal from being corrupted from transmission channel with lower channel capacity. Normally, such form of channel tends to minimize the degree of information from the reconstructed image. The contribution of this algorithm is as following-The algorithm doesn't take the complete image for compression instead it only considers a region of interest that bears utmost clinical importance and applies a wavelength coefficient to it (Line-1). The outcome is scaled (Line-2) in order to assess the size of wavelet coefficient followed by determining the scaling value $\mathrm{s}_{\mathrm{val}}$ (Line-2). If the coefficient is found to posses similar charecteristics of background (Line-3) than the algorithm performs 
scaling down the size of the coefficient by adding $\mathrm{s}_{\mathrm{val}}$ with required dimension $D$ (Line-4). This step is followed by writing the same $s_{\text {val }}$ to the bit stream (Line-5) followed by applying bit plane entropy coding i.e. $\alpha$ (Line-7). The interesting point of this algorithm is that without affecting the background, the region-ofinterest is scaled up for enhanced resolution while the background is scaled down as it is deemed nonimportant. Thecompressed image $\mathrm{x}_{3}$ therefore retains only enhanced signal information of the region-ofinterest that significant assist in forwarding the compressed signal on channel with lower channel capacity. This is now followed by further compressive sensing to enhance the signal quality as well as increase the level of optimization. The next algorithm further enhances the compression performance as shown below:

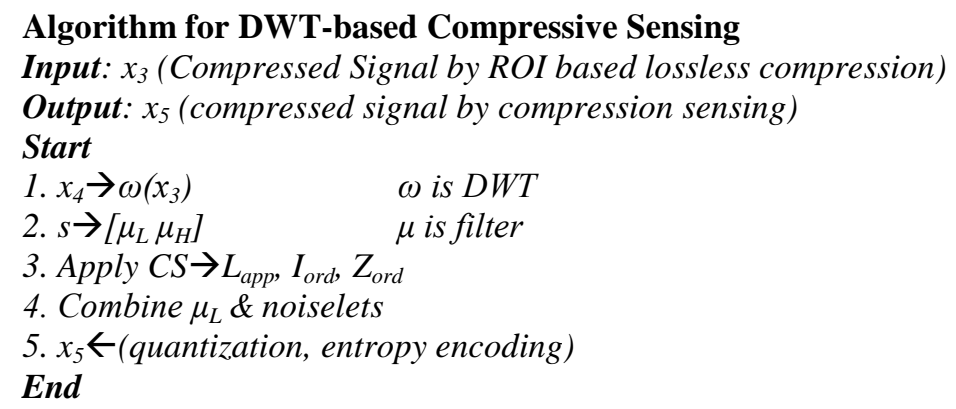

The above algorithm takes the input signal $\mathrm{x}_{4}$ from prior algorithmic step followed by applying two dimensional discrete wavelet function $\omega$ (Line-1). This results in extraction of low-pass coefficient and different auxillary a measurement that assists in compressive sensing in the next step (Line-2). The signal $s$ extracts low pass filter $\mu_{\mathrm{L}}$ and high-pass filter $\mu_{\mathrm{H}}$ (Line-2). Compressive sensing is applied over linear approximation Lapp, index ordering Iord, JPEG zigzag order Zord (Line-3) that are further combined to result using low-pass dct2 as well as noiselets (Line-4). The compressed image is obtained after performing quantization and entropy encoding. Although, an enhanced level of compression is carried out by this algorithm, we perform further optimization on it as shown by steps of next optimization algorithm:

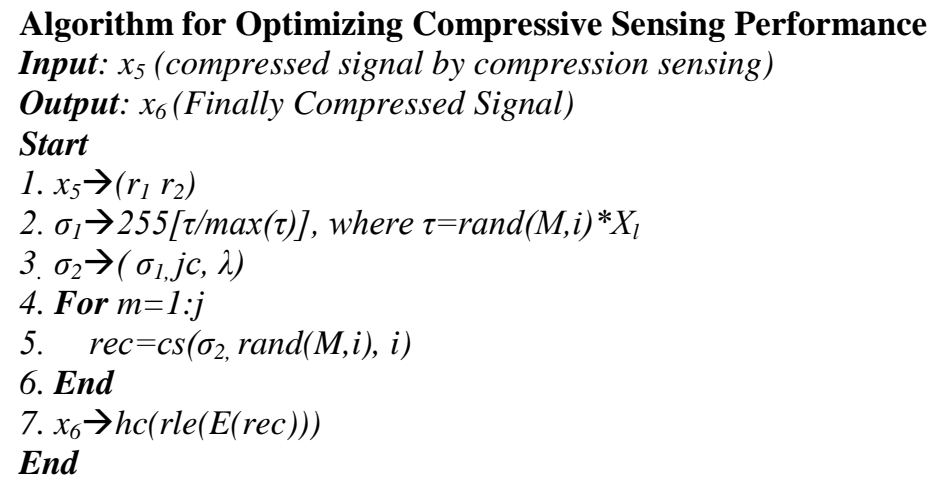

The above algorithm to perform optimization is carried out on the compressed image from prior algorithm. The algorithm considers two regions $r_{1}$ and $r_{2}$ that corresponds to clinically important and nonimportant region for a given signal (Line-1). The algorithm than computes $\tau$ that is a product of random value of $\mathrm{M}$ and another variable $\mathrm{X}_{1}$, where $\mathrm{X}_{1}$ is empirically represented by product of two dimensional row-coefficient, sparse matrix, and inverse of two dimensional row-coefficient. The optimized version of the compressed signal $\sigma_{1}$ is therefore obtained from Line- 2 which is again followed by further optimization with respect to compression ratio $\lambda$ (Line-3).

The reconstructed signal is obtained by applying compressive sensing on optimized value $\sigma_{2}$ and random value associated with $\mathrm{M}$ (Line-5). The algorithm also computes error $\mathrm{E}$ for the reconstructed image followed by run-length encoding rle (Line-7). Then Huffman coding is performed (Line-7) in order to obtain the finally compressed signal $\mathrm{x}_{6}$. It should be noted that proposed algorithm offer an extensive optimization where the complete signal is initially classified as important region $r_{1}$ and non-importat region $r_{2}$. The important region $r_{1}$ is subjected to lossless compression scheme while the non-important region $r_{2}$ consists of implying with lossy compression scheme. The lossless compression scheme is implemented using conventional JPEG2000 followed by run-length encoding and Huffman coding while the lossy compression scheme is assisted by sparsity-based transformation approach on compressive sensing. Therefore, a multilayered scheme of optimization is carried out over the compressive sensing. A sparsity-based approach is 
used for representing the signal which reduces the payload to a greater extent when the multimedia signal is allowed to be transmitted from one to another terminal. Apart from this the usage of wavelet filter performs better decomposition of signals without compromising with the informative contents within the signal. Therefore, the sparse image becomes quite computationally easier to perform compression while attempting to transmit the data over low channel-capacity network. Usage of such algorithm is highly suitable in the area of telemedicine where multiple information of medical images or video (in the form of image sequences) can be forwarded to other end using normal wireless or wired connectivity over common traffic condition. Therefore, the algorithm particularly balance the computational complexity reduction with enhancing the compressive performance.

\section{RESULTS ANALYSIS}

The implementations of the four algorithms are carried out on 1000 medical image dataset of Cornell University [35]. The dataset consists of different form of medical images (MRI image of brain, chest, leg, spine, etc) with different modalities. This section discusses both visual and numerical outcomes. Figure 2 highlights the visual outcomes where the input original image is subjected to first algorithm for extracting the random samples.

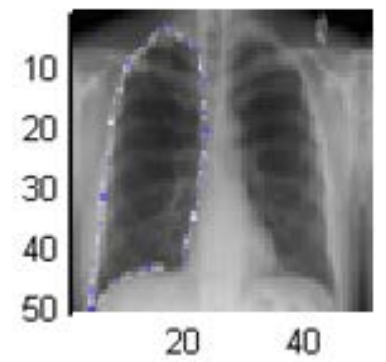

Original sample

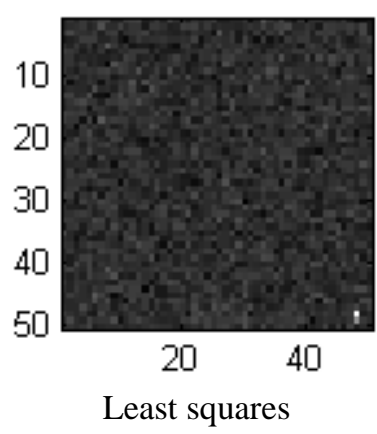

Least squares

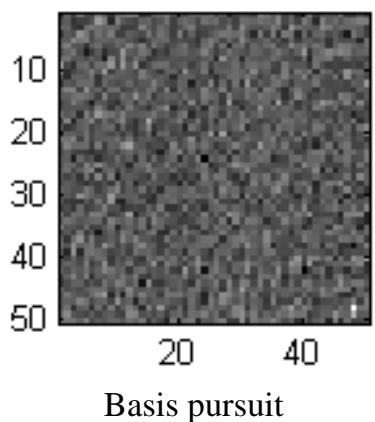

Basis pursuit

Figure 2. Visual outcomes of sampling

One of the random samples as shown in Figure 3 is the subjected to second algorithm in order to apply lossless compression scheme only. Interestingly, the visuality of the selected region of the image is unaffected by the magnification of the images as shown in Figure 3.

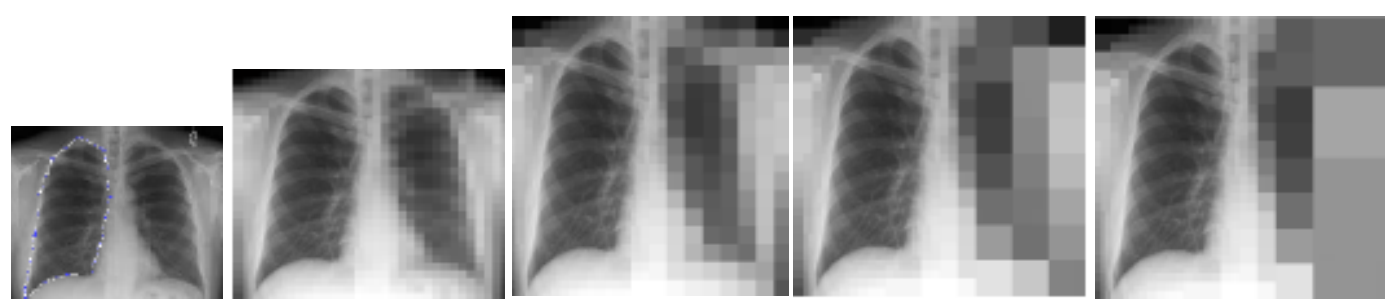

Figure 3. Visual outcomes of lossless compression

Finally, the third algorithm further enhances the signal quality followed by obtaining the reconstructed image as shown in Figure 4. We also perform a comparative performance analysis in order to assess the effectiveness of the proposed study. For tish purpose, we consider the approach discussed by Kathirvalavakumar [36], Kamargaonkar [37], Kumar [38], and Sulthana [39]. The reason of selecting the above mentioned work is the similarity of the approach using both lossy and lossless compression scheme. The existing scheme is found to use arithmetic encoding for important region and self-organizing map for non-significant region [36]. Similarly, other approaches have used Arithmatic (and Huffman encoding) on important region while the non-significant region is subjected to wavelength difference reduction, Embedded Zero Trees, and Set Partitioning in Hierarchical Trees [37]-[39]. The comparative performance analysis is 
carried out with respect to signal quality that is assessed using peak signal-to-noise ratio and algorithm processing time.

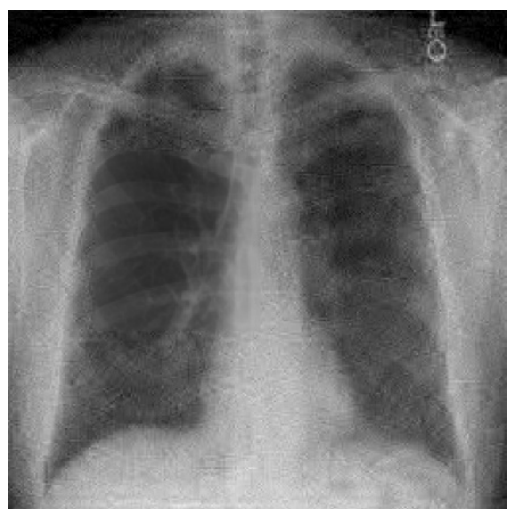

Figure 4. Visual outcomes of reconstructed image

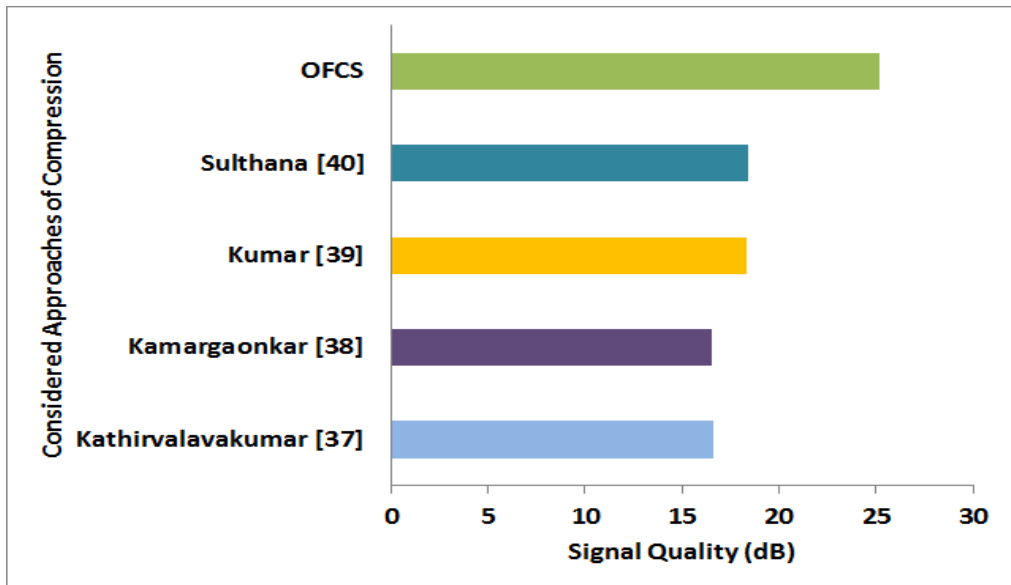

Figure 5. Comparative analysis of signal quality

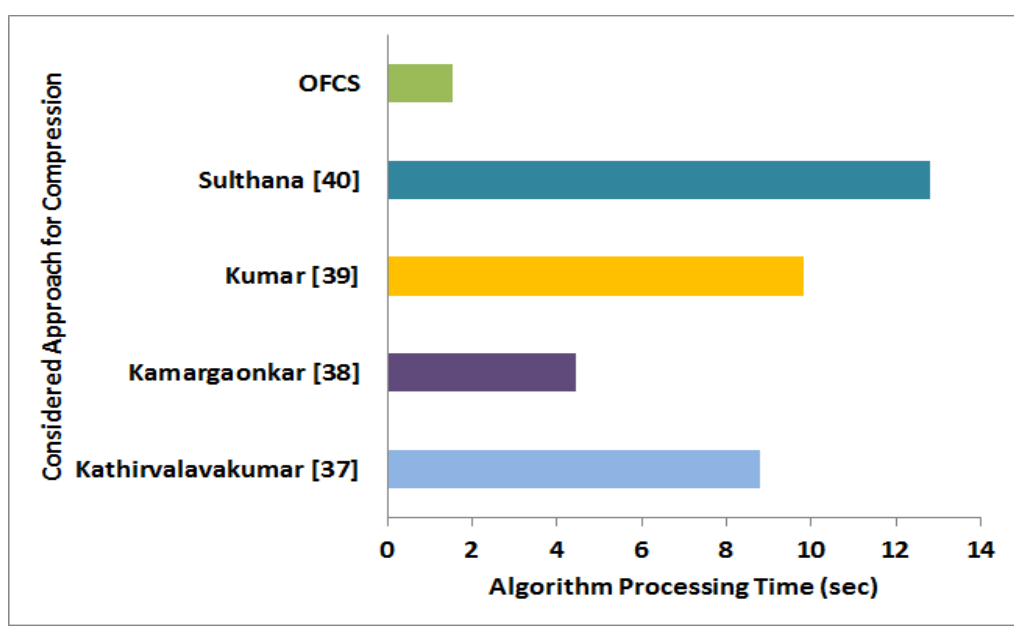

Figure 6. Comparative analysis of algorithm processing time 
The study outcome shows that proposed system offer enhanced version of signal quality and reduced algorithm processing time. The prime reason behind this outcome trend is as follows: majority of the existing system has purely emphasized on the compression size and not on the computational efficiency. Moreover, there is no much operation of optimization being carried out towards further enhancing the signal quality. On the other hand, the proposed system offers significant level of upgrading the reconstructed image signal quality exclusively focusing on the transmission over low channel capacity network.

The level of normalization technique over the processed image is too much significant as the compressive sening is applied over the sparsity of the image. The transformation techqniue using sparsity causes the complete structure of algorithm being able to disseminate the pixel information quite randomly for coping with the rate of data transmission while a balanced usage of lossy and lossless compression has resulted in optimizing the informative contents residing within the processed image. Therefore, it can be said that proposed system offers a significant solution of optimizing the compression performance to higher degree and is found to be superior in comparison to the existing system with respect to signal quality and algorithm processing time for performing compression.

\section{CONCLUSION}

Compression is very essential process for facilitating cost effective storage system as well as transmitting the medical image in telemedicine application. After reviewing various current research works, we find that the potential of compressive sensing has not been tapped properly. The existing techniques uses compressive sensing mainly for sampling process on new techniques of image acquisition. Hence, we presents a novel modeling where the goodness of lossy and lossless compression is integrated in such a balancing way that the proposed system is found to offer better compression performance. We measure the overall compression performance with an aid of PSNR and response time and found that proposed system excels much better than existing system.

\section{REFERENCES}

[1] J. Mandell, “Core Radiology: A Visual Approach to Diagnostic Imaging,” Cambridge University Press, 2013.

[2] C. Cunha and M. Manuela, "Encyclopedia of E-Health and Telemedicine,” IGI Global, 2016.

[3] D. Caramella and C. Bartolozzi, "3D Image Processing: Techniques and Clinical Applications,” Springer Science \& Business Media, 2012.

[4] R. Hoffman, "Data Compression in Digital Systems,” Springer Science \& Business Media, 2012.

[5] Lakshminarayana M. and M. Sarvagya, "Scaling the Effectiveness of Existing Compressive Sensing in Multimedia Contents,” International Journal of Computer Applications (IJCA), vol/issue: 115(9), pp. 16-26, 2015.

[6] P. J. E. Ambrosio, et al., "Image compressive sensing cryptographic analysis," 2016 International Conference on Electronics, Communications and Computers (CONIELECOMP), Cholula, pp. 81-86, 2016.

[7] S. Kavitha, et al., "Analysis and application of compressive sensing technique for rapid magnetic resonance imaging," 2016 IEEE International Conference on Recent Trends in Electronics, Information \& Communication Technology (RTEICT), Bangalore, pp. 405-408, 2016.

[8] M. T. Bevacqua, et al., "Wave atom based Compressive Sensing and adaptive beamforming in ultrasound imaging,” 2015 IEEE International Conference on Acoustics, Speech and Signal Processing (ICASSP), South Brisbane, QLD, pp. 2474-2478, 2015. tic Nanoparticles as Contrast Agent, in IEEE Transactions on Medical Imaging, vol/issue: 35(2), pp. 665-673, 2016.

[9] F. Foroozan and P. Sadeghi, "Wave atom based Compressive Sensing and adaptive beamforming in ultrasound imaging," 2015 IEEE International Conference on Acoustics, Speech and Signal Processing (ICASSP), South Brisbane, QLD, pp. 2474-2478, 2015.

[10] Z. Chen, et al., "Compressive Deconvolution in Medical Ultrasound Imaging," in IEEE Transactions on Medical Imaging, vol/issue: 35(3), pp. 728-737, 2016.

[11] L. Guo and A. M. Abbosh, "Compressive sensing for stroke detection in microwave-based head imaging," 2014 IEEE Antennas and Propagation Society International Symposium (APSURSI), Memphis, TN, pp. 1911-1912, 2014.

[12] L. Baldassarre, et al., "Learning-Based Compressive Subsampling," in IEEE Journal of Selected Topics in Signal Processing, vol/issue: 10(4), pp. 809-822, 2016.

[13] B. Jelena, "Comparison of algorithms for compressed sensing of magnetic resonance images," 2015 4th Mediterranean Conference on Embedded Computing (MECO), Budva, pp. 303-306, 2015.

[14] J. Yang, et al., "A novel fusion technique for CT and MRI medical image based on NSST,” 2016 Chinese Control and Decision Conference (CCDC), Yinchuan, pp. 4367-4372, 2016.

[15] T. Küstner, et al., "MR Image Reconstruction Using a Combination of Compressed Sensing and Partial Fourier Acquisition: ESPReSSo,” in IEEE Transactions on Medical Imaging, vol/issue: 35(11), pp. 2447-2458, 2016.

[16] Delai L., et al., "The application of compressive sensing based on wavelet in the reconstruction of ultrasonic medical image," 2011 Second International Conference on Mechanic Automation and Control Engineering, Hohhot, pp. 5350-5353, 2011. 
[17] M. Panić, et al., "Compressed sensing in MRI with a Markov random field prior for spatial clustering of subband coefficients,” 2016 24th European Signal Processing Conference (EUSIPCO), Budapest, pp. 562-566, 2016.

[18] Y. Sa, "Medical Image Registration Algorithm Based on Compressive Sensing and Scale-Invariant Feature Transform,” 2015 8th International Conference on Intelligent Computation Technology and Automation (ICICTA), Nanchang, pp. 547-551, 2015.

[19] S. Sharma, et al., "A New Sparse Signal-Matched Measurement Matrix for Compressive Sensing in UWB Communication,” in IEEE Access, vol. 4, pp. 5327-5342, 2016.

[20] X. Xu, et al., "Adaptive Hierarchical Data Aggregation using Compressive Sensing (A-HDACS) for Non-Smooth Data Field,” 2014 IEEE International Conference on Communications (ICC), Sydney, NSW, pp. 65-70, 2014.

[21] Y. Wang, et al., "Data-Driven Sampling Matrix Boolean Optimization for Energy-Efficient Biomedical Signal Acquisition by Compressive Sensing," in IEEE Transactions on Biomedical Circuits and Systems, vol/issue: 11(2), pp. 255-266, 2017.

[22] Q. Zhang, et al., "Adaptive sampling and wavelet tree based compressive sensing for MRI reconstruction,” 2016 IEEE International Conference on Image Processing (ICIP), Phoenix, AZ, pp. 2524-2528, 2016.

[23] Y. Zhang, et al., "A Novel Compressed Sensing Method for Magnetic Resonance Imaging: Exponential Wavelet Iterative Shrinkage-Thresholding Algorithm with Random Shift,” Hindawi Publishing Corporation, International Journal of Biomedical Imaging, 2016.

[24] C. Zhao, et al., "Video Compressive Sensing Reconstruction via Reweighted Residual Sparsity," in IEEE Transactions on Circuits and Systems for Video Technology, vol/issue: 27(6), pp. 1182-1195, 2017.

[25] Y. Xi, et al., "Simultaneous CT-MRI Reconstruction for Constrained Imaging Geometries Using Structural Coupling and Compressive Sensing," in IEEE Transactions on Biomedical Engineering, vol/issue: 63(6), pp. 13011309, 2016.

[26] H. Mesra, et al., "New Lossless Compression Method using Cyclic Reversible Low Contrast Mapping (CRLCM)," International Journal of Electrical and Computer Engineering (IJECE), vol/issue: 6(6), pp. 2836-2845, 2016.

[27] E. Setyaningish and A. Harjoko, "Survey of Hybrid Image Compression Techniques," in International Journal of Electrical and Computer Engineering (IJECE), vol/issue: 7(4), pp. 2206-2214, 2017.

[28] Suma and V. Sridhar, "Design of Multiplier for Medical Image Compression Using Urdhava Tiryakbhyam Sutra," International Journal of Electrical and Computer Engineering (IJECE), vol/issue: 6(3), pp. 1140-1151, 2016.

[29] Lakshminarayana M. and M. Sarvagya, "Lossless Compression of Medical Image to Overcome Network Congestion Constraints,” SPRINGER-AISC-ERCICA-2015, NMIT, Bangalore, vol. 01, pp. 305-311, 2015.

[30] Lakshminarayana M. and M. Sarvagya, "Random Sample Measurement and Reconstruction of Medical Image Signal using Compressive Sensing,” IEEE-CoCoNet'15, IIITM-K, Technopark, Trivandrum, Kerala, pp. 261-268, 2015.

[31] Lakshminarayana M. and M. Sarvagya, “Algorithm to Balance Compression and Signal Quality using Novel Compressive Sensing in Medical Images,” SPRINGER-CSoC-2016, Vol.465, Tomas Bata University in Zlin, Czech Republic, pp. 317-327, 2016.

[32] Lakshminarayana M. and M. Sarvagya, "HAMIC: A Novel Modelling of Hybrid Algorithm for Medical Image Compression,” IEEE-INDIACom-2017, Bharati Vidyapeeth, New Delhi, 2017.

[33] Lakshminarayana M. and M. Sarvagya, "CARIC: A Novel Modeling of Combinatorial Approach for Radiological Image Compression,” SPRINGER-CSoC-2017, Vol. Tomas Bata University in Zlin, Czech Republic, 2017.

[34] Lakshminarayana M. and M. Sarvagya, "RM2IC: Performance Analysis of Region based Mixed-mode Medical Image Compression,” International Journal of Image, Graphics and Signal Processing (IJIGSP), vol/issue: 9(10), pp. 12-21, 2017.

[35] "Finding Articles, Databases and Images," Cornell University Library. https://www.library.cornell.edu/research/introduction/articles.

[36] T. Kathirvalavakumar and E. Ponmalar, "Self-organizing map and wavelet based image compression,” SpringerInternational Journal of machine Learning and Cybernetics, 2012.

[37] C. Kamargaonkar and M. Sharma, "Hybrid medical image compression method using SPIHT algorithm and haar wavelet transform," IEEE International Conference on Electrical, Electronics, and Optimization Techniques, 2016.

[38] R. Kumar, et al., "Electrocardiogram signal compression using singular coefficient truncation and wavelet coefficient coding,” IEEE- IET Science. Measurement \& Technology, 2015.

[39] S. N. Sulthana and M. Chandra, "Image compression with adaptive arithmetic coding," IJCA Online, vol/issue: 1(18), pp. 35-37, 2010. 


\section{BIOGRAPHIES OF AUTHORS}

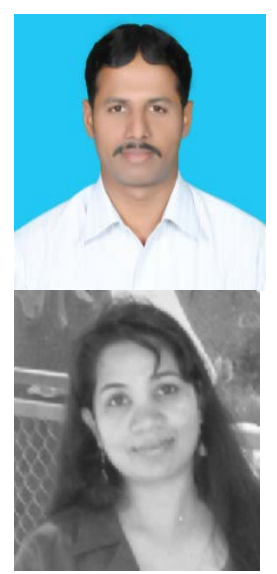

Lakshminarayana. M received the B.E. degree in Medical Electronics from Dr. AIT, Bangalore in 2005 and the M. Tech degree in Electronics from Sir. MVIT, Bangalore in 2007 under Visvesvaraya Technological University, Belgaum, where he is currently working towards the Ph.D. degree under VTU in the field of Multimedia Communication applications. He is the member of Professional bodies IEEE, MISTE, IAENG. His current research interests include the areas of Multimedia communication, Image and video processing and DSP applications in embedded system design.

Dr. Mrinal Sarvagya, Professor, School of Electronics and Communication Engineering PhD degree in Wireless Communication from IIT Kharagpur, MTech degree in digital Communication, (Course work from IIT kanpur) from DAVV Indore and B.E degree in Electronics and communication Engineering ” from Govt Engineering College Ujjain. ”. She received the best thesis award from IIT Kharagpur in the year 2009 for her thesis titled "QoS based packet scheduling and resource allocation schemes for WCDMA UMTS". She is the member of Professional bodies WIE, IEEE. He/She has 15 years of teaching experience, teaching various subjects like Wireless communication, Advanced Digital Communication, Computer communication and networking, real time Operating Systems, Adhoc wireless networks, Protocol Engineering. Her area of research is Wireless communication, channel equalization in OFDM-IDMA / SCM receivers, Cognitive Radio networks. Principal Investigator, VGST Karnataka "Zero padding OFDM signals for Cognitive radio Networks" (2012 -2014). Principal Investigator, IEEE Hyderabad "Multimedia Communication over Fibre Optical link" (2012) Installed at Birla science Museum Hyderabad. Currently Principal Investigator for the sponsored Project from Naval research Board, Ministry of defence India (2015). 